\title{
Differences in Amount of Informal Care Received by Non-Hispanic Whites and Latinos in a Nationally Representative Sample of Older Americans
}

\author{
Carlos O. Weiss, MD, ${ }^{*}$ Hector M. González, PhD, ${ }^{\dagger}$ Mohammed U. Kabeto, MS, ${ }^{\ddagger}$ and \\ Kenneth M. Langa, MD, PhD ${ }^{\ddagger \mathcal{S} \|}$
}

The objective of this study was to evaluate informal (unpaid) care and its broad determinants for Latinos in a nationally representative sample. A cross-sectional analysis of the 1993 Asset and Health Dynamics Study, a national probability sample of 7,443 older adults aged 70 and older, was performed to determine the independent effect of Latino ethnicity on the receipt of informal care by disabled older individuals. Self-reported race/ethnicity was used to predict the mean daily hours of informal care received for activity of daily living (ADL) or instrumental activity of daily living (IADL) assistance after adjustment for predisposing, need, and enabling variables. There was a significant association between informal home care and ethnic group, with $44.3 \%$ of Latinos receiving informal care, compared with $33.9 \%$ of African Americans and $24.6 \%$ of non-Hispanic whites $(P<.001)$. After adjustment, Latinos received 11.0 weekly hours of informal care, compared with 7.5 hours for non-Hispanic whites and 6.3 hours for African Americans $(P<.001)$. The results from this nationally representative sample indicate that Latinos receive significantly more hours of informal care on average than African Americans or non-Hispanic whites for ADL and IADL disability. Clinicians should be alert to the significant

From the *enter on Aging and Health, Bloomberg School of Public Health, Johns Hopkins University, Baltimore, Maryland; 'Department of Epidemiology, School of Public Health, ${ }^{\ddagger}$ Division of General Medicine, School of Medicine, and ${ }^{\S}$ Institute for Social Research, University of Michigan, Ann Arbor, Michigan; and "Center for Practice Management and Outcomes Research, Department of Veterans Affairs, Ann Arbor, Michigan.

The National Institute on Aging provided funding for the Asset and Health Dynamics Study (U01 AG09740), data from which were used in this analysis. An abstract of this work was presented as a poster at the American Geriatrics Society Annual Scientific Meeting in Baltimore, Maryland, May 14-18, 2003. Dr. Weiss received support from a National Institute on Aging Training Grant (T32AG00247) during the completion of this work. Dr. González was supported by a Career Development Award from the National Institute on Mental Health (K08 MH67726). Dr. Langa was supported by a Career Development Award from the National Institute on Aging (K08 AG19180), a New Investigator Research Grant from the Alzheimer's Association and a Paul Beeson Physician Faculty Scholar in Aging Research Award.

Address correspondence to Carlos O. Weiss, MD, Center on Aging and Health, 2024 E. Monument St., Suite 2-700, Baltimore, MD 21205.

E-mail: cweiss9@jhmi.edu amount of informal care and possible associated strain in caregivers of older Latinos. J Am Geriatr Soc 53: 146-151, 2005.

Key words: social support; Latino; disabled persons

B 2030, the U.S. population of Latinos is projected to increase to $19.4 \%$ and account for a significant portion of the coming boom in the older population in this country. ${ }^{1}$ Older Latinos have a high burden of some diseases and poor self-perceived health. ${ }^{2}$ They are also likely to be at higher risk for poor health outcomes due to higher rates of poverty and lower rates of health insurance coverage than non-Hispanic Whites (NHWs). ${ }^{3}$ For these reasons, understanding the use of informal sources of health care in this growing population is important to clinicians, healthcare managers, and policy makers, yet currently little is known about patterns in informal care (unpaid help from family and friends) of disabled older Latinos in the United States at the population level.

Although some reports have suggested that Latinos receive more informal care (unpaid help from family and friends) than NHWs, the data supporting the premise that Latinos are more traditional in their family structure and provide more care as a group are limited. ${ }^{4} \mathrm{An}$ analysis of the Channeling Study using 1982 to 1984 data found that Latinos and African Americans (AAs) used more informal care than NHWs, ${ }^{5}$ but the fact that the majority of Latinos it included were Cuban Americans living in Florida limited that study. More is known about patterns of utilization of formal (paid) healthcare. For example, patterns of formal care utilization found in the Channeling Study indicate that Latinos used less formal care than NHWs, but they used more organized community-based services such as congregate meals and counseling. ${ }^{6}$ In general, the limited literature on informal care of Latinos, quantitative and qualitative, has used nonrepresentative samples..$^{7-12}$

Given the growing number of elderly Latinos and the current lack of nationally representative information about 
this population, this study's objective was to obtain estimates of the amount of informal care provided to this group and to better identify and understand its broad determinants. This study used an approach that organized variables affecting healthcare utilization into predisposing, enabling, and need factors. ${ }^{13}$ Predisposing variables include sociodemographic characteristics such as age, sex, and level of education. Enabling variables refer to social and monetary resources at the level of the family (income, insurance) and the community (e.g., physicians and hospital beds per capita). Need variables include the level of disability, the use of other healthcare services, and recent hospitalizations. This conceptual outline is most often used to model the utilization of formal healthcare services. It is employed here as an organizing framework for the "utilization" of informal care because of its familiarity and because categories of enabling, need, and predisposing variables will influence the receipt of informal care as well. Comparison was made with NHWs and AAs, but given space limitations and because there is less information on Latinos, the discussion focuses on this group.

\section{METHODS}

\section{Participants and Measures}

Data from the 1993 Asset and Health Dynamics (AHEAD) study, a nationally representative probability sample of community-dwelling-older adults aged 70 and older $(\mathrm{N}=7,443)$ were used. AHEAD was designed to collect information on health, functional status, and care provided by paid and unpaid caregivers in the home using telephone and in-person interviews. Most respondents aged 70 to 79 $(75.2 \%)$ were interviewed by telephone. Most of those aged 80 and older $(63.2 \%)$ were interviewed in person. The overall survey response rate was $80.4 \%$, and response rate did not differ significantly between telephone and in-person interviews. For both forms of interview, respondents selected English or Spanish for communication. To minimize attrition, proxy respondents were interviewed in cases in which the selected respondents were unable to answer survey questions because of physical illness, cognitive impairment, or other reasons $\left(10.4 \%\right.$ overall). ${ }^{14}$

Respondents were classified as informal care recipients if "because of a health problem" they received in-home assistance with any activity of daily living (ADL) or instrumental activity of daily living (IADL) from a paid or unpaid relative or an unpaid person with no organizational affiliation. ${ }^{15}$ Due to the survey design, if care for an ADL item was received most of the time, respondents were asked further questions. If the response was only some of the time or occasionally, they were not. In contrast, for IADL items, any help triggered questions about the amount of care received. Respondents were asked, "In the last month how often did (your helper) help you?" If the response was "about once a week" or more, they were asked "On the days (your helper) helped you, about how many hours per day was that?" 16 Because data on hours per day of care were not collected for caregivers who helped less than once per week, weekly hours of care for these infrequent helpers were imputed using a regression model based on reported caregiver characteristics (helper sex, residential status, relationship to the respondent, and number of days per week of care). ${ }^{16,17}$
This occurred for less than $2 \%$ of the total informal caregiver hours analyzed in the study, and the likelihood of missing data did not differ significantly across ethnic groups. All analyses were repeated after dropping any observation for which data were imputed, and there was no significant change in the results. A limit of 16 hours of care per day for any individual caregiver was imposed to allow for 8 hours of sleep, affecting only $6.2 \%$ of the 2,700 informal caregivers identified in the AHEAD study.

All respondents were asked, "Do you consider yourself Hispanic or Latino?" and "Do you consider yourself primarily white or Caucasian, black or African American, American Indian, or Asian, or something else?" If a respondent answered yes to the first question, he or she was categorized as Hispanic or Latino. This group was then asked if they were Mexican American/Chicano, Puerto Rican, Cuban American, or other Latino. Because of small sample sizes, subgroup analyses of the Latino group were not possible. Ninety-eight respondents in the American Indian, Asian, or other categories were excluded, so the final sample included 7,345 respondents classified as NHW, AA, or Latino.

The following predisposing measures were included in the analyses: age group $(70-79,80-89, \geq 90)$, sex, living arrangement (married, unmarried living with someone, unmarried living alone), education (grades 0-11, high school, some college, college graduate), and place of birth. Variables reflecting healthcare need were level of disability, based on ADL and IADL limitations (grouped ADL 0, 1-3, 4-6 and IADL $0,1-3,4-5)$, self-reported chronic medical conditions (diabetes mellitus, heart disease, hypertension, stroke, cancer, lung disease, psychiatric problem, arthritis, and incontinence), cognitive impairment defined using a validated cognitive screening test adapted from the Telephone Interview for Cognitive Status, ${ }^{18}$ hospitalization within the previous year, and nursing home stay within the previous year. Enabling variables were economic status by household net worth $($ low $(<\$ 38,000)$, middle $(\$ 38,000$ $139,000)$, and high $(>\$ 139,000)$ tertiles), insurance (Medicare, Medicaid, supplemental), region of residence (east, central, south, or west), and living in a metropolitan statistical area.

\section{Statistical Analyses}

A standard two-part multivariate regression model was used to identify the independent association between ethnicity and hours of informal care. ${ }^{5,19,20}$ To determine the likelihood of receiving informal home care, logistic regression was used first to estimate the association between informal home care and ethnicity while controlling for the other covariates. In the second part of the analysis, ordinary least squares regression was used to examine the association between the natural log of informal care hours and ethnicity for respondents who received care. Then the predictions for each ethnic category from the logistic regression and the ordinary least squares regression were combined to obtain an estimate of the average effect of ethnicity on weekly hours of informal care. All analyses were weighted and adjusted for the complex sampling design of AHEAD.

Three separate regression models were used. The first model controlled for basic sociodemographic, or 
predisposing, variables (age, sex, living arrangement, education, and place of birth). The second included the variables from Model 1 and need variables (level of disability, presence of chronic illnesses, and recent nursing home or hospital stay). The third analysis included the variables from Model 2 and enabling variables (net worth, Medicare, Medicaid and supplemental insurance, region of residence, and residence in a metropolitan statistical area). By performing this staged analysis, the influence of different categories of variables could be better identified. In the last analysis, the remaining informal care attributable to ethnicity is presumably independent of the other predisposing, need, and enabling variables.

\section{RESULTS}

The characteristics of the study population $(\mathrm{N}=7,345)$ are shown in Table 1 . About $5.7 \%$ of respondents self-identified themselves as Latino, $13.8 \%$ as AA, and $80.5 \%$ as NHW. About $58.5 \%$ of Latino respondents were Mexican American. Approximately half of the rest of the Latino respondents were Cuban American; one-quarter were Puerto Rican, Spanish, or Central or South American; and onequarter were other. Most Latino respondents (56.3\%) preferred to respond in Spanish. There were no significant age or sex differences across ethnic groups. Latinos were less likely to be married than NHWs or AAs $(P<.001)$. Older AAs and Latinos had more ADL and IADL limitations than NHWs $(P<.001)$ and had significantly higher rates of cognitive impairment $(P<.001)$, diabetes mellitus $(P<.001)$, and arthritis $(P<.001)$ but lower rates of heart disease $(P=.003)$ and cancer $(P=.001)$. Latino respondents were most likely to report a psychiatric problem $(P=.02)$ and had the lowest net worth $(P<.001)$ and highest rate of Medicaid coverage $(P<.001)$. There was no significant difference across groups with respect to nursing home or hospital admission within the previous year. There was a significant association between the likelihood of receiving informal care and ethnic group $(P<.001)$, with $44.3 \%$ of Latinos, $33.9 \%$ of AAs, and $24.6 \%$ of NHWs receiving informal care.

Table 2 shows the unadjusted weekly hours of informal care received by ethnic category and the adjusted hours derived from the regression models. Older Latinos received significantly more weekly hours of care than AAs and NHWs in all models. For Latinos, this amounted to 11 weekly hours of care, compared with approximately 7.5 hours for NHWs and 6.3 hours for AAs, after controlling for all variables. After adjusting for predisposing variables (Model 1), the difference between the amount of informal care received by Latinos and NHWs decreased significantly, from approximately 8.5 to 4.9 average hours per week. Further controlling for need variables (Model 2) decreased the differential only slightly, from 4.9 to 3.6 hours. Additional controls for enabling variables (Model 3) resulted in almost no additional changes across ethnic categories.

\section{DISCUSSION}

This is the first study to the authors' knowledge to examine ethnicity and informal care in a large, nationally representative sample of older adults with adequate controls for variables that might influence the receipt of health care.
More older Latino individuals $(44.3 \%)$ received informal care than AAs (33.9\%) or NHWs $(24.6 \%)$ in this sample. Latinos also received significantly more weekly hours of informal care, on average, than AAs or NHWs (11.0 vs 6.3 and 7.5 weekly hours, respectively).

Disabled older Latinos received more hours of informal care even after controlling for a wide range of other variables. What factors might explain this group difference? Although many variables undoubtedly influence the relationship between Latino ethnicity and informal care, the findings reported here suggest that need and enabling factors, such as level of disability and disease and socioeconomic status, do not entirely explain the observed differences in the amount of informal care across ethnic groups. Differences in psychosocial predisposing factors related to ethnicity, such as cultural values and norms regarding responsibilities toward elders, probably play a significant role in the amount of informal care received. ${ }^{12}$

This study can also inform clinicians in caring for older Latino individuals and their families. Heterogeneity with respect to health knowledge and attitudes among Latinos is high, making overgeneralization using racial or ethnic identification a possibility, but it might be suggested that care teams gather information about the amount and type of informal care received by older Latinos because it will help in implementing appropriate individualized treatment plans. Doing so without preconceptions might provide an important opportunity to gain understanding of culturally related health beliefs within Latino families and might foster improved communication between physicians, patients, and caregivers. The finding that disabled older Latinos received significantly greater amounts of informal care than other ethnic groups in this study should alert clinicians to the potential for increased burden on caregivers for these individuals, especially given recent findings that Latino caregivers for patients with dementia have a significantly higher risk of depression than NHW or AA caregivers. ${ }^{21}$

A closer examination of predisposing variables in this study reveals interesting findings. There were no significant differences in age or sex distribution across ethnic groups, making it unlikely that these variables significantly influenced these results. Prior studies have shown that living arrangement and available caregiver network can play a significant role in the receipt of informal care. ${ }^{11}$ Although it is often assumed that older Latinos live in common with many family members, the majority of older Latinos $(71 \%)$ do not live with extended families, ${ }^{22}$ and in this sample, a significant proportion of older Latinos $(28.1 \%)$ lived alone. The final estimates of weekly hours of informal care that are reported here were adjusted for this difference.

A major contribution of this study is the examination of covariates that can confound the role of ethnicity. Many prior studies have suggested the importance of need variables in identifying those who use greater amounts of home care. For example, cross-sectional analyses of the Channeling Study ${ }^{5,6}$ and longitudinal analyses of the National Long-Term Care Survey ${ }^{23}$ and pooled Health Interview Surveys ${ }^{10}$ supported the importance of level of disability.

Prior research has also shown that cognitive impairment, which is particularly associated with need for supervision and assistance with ADLs, was associated with significantly more hours of informal care. ${ }^{17}$ Similarly, in an 
Table 1. Distribution of Characteristics of Respondents by Ethnic Category $(\mathrm{N}=7,345)$

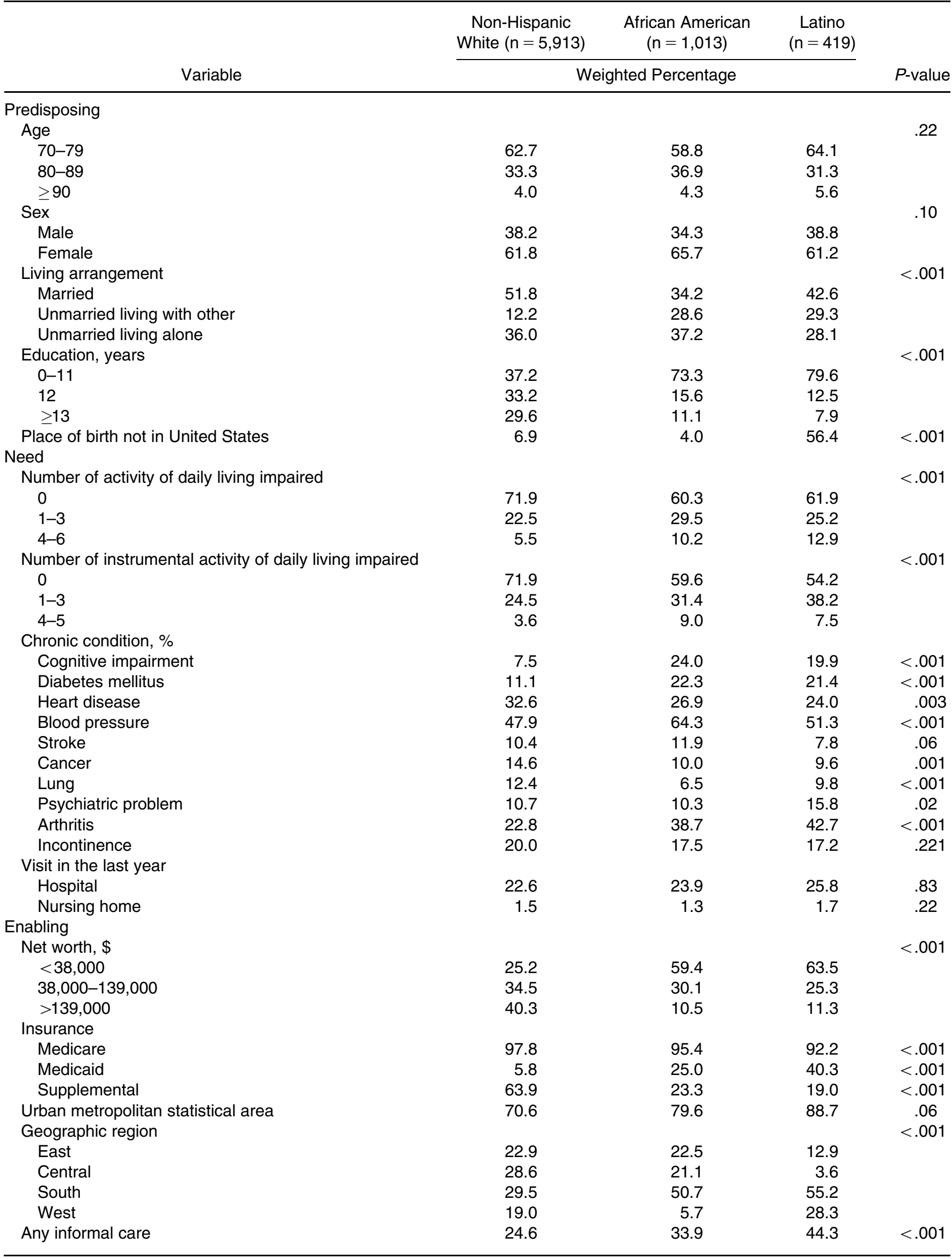


Table 2. Adjusted Average Weekly Informal Care by Ethnic Category

\begin{tabular}{lcccc}
\hline & Unadjusted & Model 1* & Model 2 $^{\dagger}$ & Model $^{\ddagger}$ \\
\cline { 2 - 4 } \multicolumn{1}{c}{ Ethnic Category } & \multicolumn{4}{c}{ Hours $(95 \%$ Confidence Interval) } \\
\hline Non-Hispanic White & $6.0(5.2-6.7)$ & $6.6(6.1-6.6)$ & $7.4(6.8-8.0)$ & $7.5(6.9-8.0)$ \\
African American & $10.2(7.6-12.8)$ & $7.7(7.4-7.9)$ & $6.4(5.9-6.9)$ & $6.3(5.8-6.8)$ \\
Latino & $14.5(11.7-17.3)$ & $11.3(11.0-11.7)$ & $11.0(10.2-11.8)$ & $11.0(10.2-11.8)$ \\
\hline
\end{tabular}

* Adjusted for age, sex, living arrangement, education, and place of birth.

${ }^{\dagger}$ Adjusted for variables in Model 1 plus nursing home, hospital, ADLs, IADLs, and chronic conditions, including cognitive impairment.

${ }^{\ddagger}$ Adjusted for variables in Model 2 plus net worth, Medicare, Medicaid, supplemental insurance, region of country, and urban versus rural residence.

ADLs $=$ activities of daily living; IADLs $=$ instrumental ADLs.

exploratory analysis using the same data, a significantly greater quantity of informal care was detected in individuals with cognitive impairment, even after controlling for all other variables (data not shown). This is important in light of recent prevalence estimates indicating that Latinos are at equal or greater risk of dementia than $\mathrm{NHWs}^{24,25}$ and emphasizes the importance of increasing efforts to adequately prevent and treat cognitive impairment in the Latino community. ${ }^{26}$ It has become clear that controlling for differences in the prevalence of cognitive impairment across ethnic groups is important when trying to understand and interpret ethnic differences in informal care and other healthcare utilization. The final estimates of receipt of informal care presented here are adjusted for the presence of cognitive and functional impairment.

In this analysis, net income and insurance availability did not have independent effects on the amount of informal care received. Similarly, it has been shown that race was not a significant predictor of the use of formal long-term care services after controlling for income. ${ }^{28}$

This study found that AAs received significantly fewer hours of informal care than Latinos. The models suggest that differences across the groups in need variables contributed to the difference in hours of informal care received, but there are likely other important differences in social support, such as primary caregiver versus shared caregiving arrangements, which remain to be more fully explored. ${ }^{4,29}$ Differences in English proficiency affecting informal care indirectly through less access to formal care may also play a role in this difference.

A number of potential limitations should be considered when interpreting the results of this study. Small sample sizes made it impossible to assess Latino subgroup heterogeneity in Puerto Ricans, Cuban Americans, and other Latinos. In a secondary analysis (data not shown), a categorical variable for year of arrival to the United States (e.g., 1900-1919, 1920-1939) was added to the categorical variable on nativity already in the model as a crude indicator of acculturation, and Mexican Americans were separated from other Latinos. The findings were consistent with a larger difference in hours of informal care received specifically by Mexican Americans than in the main results. At the population level, the Hispanic Health and Nutrition Examination Survey of 1982 to 1984 found that Mexican Americans differed from other Latino subgroups in access to health insurance and use of preventive services. ${ }^{30}$ This may have been because, on average, Mexican Americans were less likely to have health insurance than Puerto Ricans (who are U.S. citizens and eligible for federal insurance programs) and Cuban Americans (who in general have higher incomes and better access to employee health insurance). In the future, a combination of focused ethnographic studies and cohort studies containing large numbers within Latino subgroups could shed more light on the question of variation within this group. ${ }^{12}$ Because the AHEAD study did not collect information on acculturation or knowledge and attitudes regarding health care, this analysis did not directly test the idea that knowledge and attitudes play a central role in how much informal care is provided among ethnic groups. It should also be noted that these data were collected in 1993 to 1994. Large secular changes in the relationships between the amount of care provided and ethnicity studied here are unlikely over the intervening period, but it is possible that these data do not reflect current care patterns. Another important question that this study did not address was whether Latinos received more help than they needed or whether the other groups received less help than they needed. Also, longitudinal analyses will be necessary to elucidate more specific cause-and-effect relationships and interactions, as well as cohort effects, which may be expected over time.

As with all observational studies, the possibility exists that a variable omitted from the analysis (e.g., another comorbidity or socioeconomic status variable) that is correlated with Latino ethnicity and informal care is the true cause of the greater amount of informal care that was found in Latinos, but many important sociodemographic measures, functional limitations, and the common comorbidities that have previously been shown to influence the level of informal care in the elderly were included, so the finding of increased amounts of informal care by Latinos appears to be well supported.

The methods used in the AHEAD study are likely to lead to conservative estimates of the amount of informal care received. Only information on help received for ADLs or IADLs was requested, so assistance provided for some important activities is not represented. Respondents were instructed to consider assistance received due to health problems, thus receipt of aid not perceived as related to a limitation imposed by illness was not included in the analysis. Finally, individuals were asked questions about amount of care received for ADLs only if they received help "most of the time."

This study of a nationally representative sample of older Latinos shows that they receive significantly more informal care than AAs and NHWs. The findings reported 
here suggest that ethnicity remains a central factor in the receipt of informal care, even after careful adjustment for enabling and need variables. The reasons for this are complex, but these results indicate that further examination of psychosocial predisposing factors in ethnic groups will be a source of important information. Future studies exploring the dynamics of disablement and caregiving over time might explain why older Latinos received a greater number of informal care hours, and therefore aid in meeting the needs of the growing older Latino population.

\section{ACKNOWLEDGMENT}

The authors would like to thank the anonymous reviewers for their helpful comments.

\section{REFERENCES}

1. U.S. Bureau of the Census. Projections of the Resident Population by Race, Hispanic Origin, and Nativity: Middle Series, 2025-2045. [on-line]. Available at: www.census.gov/population/projections/nation/summary/np-t5-f.txt. Accessed December 2, 2003.

2. Bassford TL. Health status of Hispanic elders. Clin Geriatr Med 1995;11: 25-38.

3. Angel RJ, Angel JL, Markides KS. Stability and change in health insurance among older Mexican Americans: Longitudinal evidence from the Hispanic established populations for epidemiologic study of the elderly. Am J Public Health 2002;92:1264-1271.

4. Navaie-Waliser M, Feldman PH, Gould DA et al. The experiences and challenges of informal caregivers: Common themes and differences among whites, blacks, and Hispanics. Gerontologist 2001;41:733-741.

5. Kemper P. The use of formal and informal home care by the disabled elderly. Health Serv Res 1992;27:421-451.

6. Mui AC, Burnette D. Long-term care service use by frail elders: Is ethnicity a factor? Gerontologist 1994;34:190-198.

7. Wallace SP, Campbell K, Lew-Ting CY. Structural barriers to the use of formal in-home services by elderly Latinos. J Gerontol 1994;49:S253-S263.

8. Wallace SP, Levy-Storms L, Ferguson LR. Access to paid in-home assistance among disabled elderly people. Do Latinos differ from non-Latino whites? Am J Public Health 1995;85:970-975.

9. Wallace SP, Levy-Storms L, Kington RS et al. The persistence of race and ethnicity in the use of long-term care. J Gerontol B Psychol Sci Soc Sci 1998;53B:S104-S112.

10. Wolinsky FD, Aguirre BE, Fann LJ et al. Ethnic differences in the demand for physician and hospital utilization among older adults in major American cities: Conspicuous evidence of considerable inequalities. Milbank Q 1989;67: 412-449.
11. Burton L, Kasper J, Shore A et al. The structure of informal care: Are there differences by race? Gerontologist 1995;35:744-752.

12. Dilworth-Anderson P, Williams IC, Gibson BE. Issues of race, ethnicity, and culture in caregiving research: A 20-year review (1980-2000). Gerontologist 2002;42:237-272.

13. Bradley EH, McGraw SA, Curry L et al. Expanding the Andersen model. The role of psychosocial factors in long-term care use. Health Serv Res 2002; 37:1221-1242.

14. Soldo BJ, Hurd MD, Rodgers WL et al. Asset and health dynamics among the oldest old: An overview of the AHEAD Study. J Gerontol B Psychol Sci Soc Sci 1997;52B Spec No:1-20.

15. Norgard TM, Rodgers WL. Patterns of in-home care among elderly black and white Americans. J Gerontol B Psychol Sci Soc Sci 1997;52B Spec No: 93-101.

16. Wolf DA, Freedman V, Soldo BJ. The division of family labor: Care for elderly parents. J Gerontol B Psychol Sci Soc Sci 1997;52B Spec No: 102-109.

17. Langa KM, Chernew ME, Kabeto MU et al. National estimates of the quantity and cost of informal caregiving for the elderly with dementia. J Gen Intern Med 2001;16:770-778.

18. Herzog AR, Wallace RB. Measures of cognitive functioning in the AHEAD Study. J Gerontol B Psychol Sci Soc Sci 1997;52B Spec No: 37-48.

19. Duan N, Manning WG, Morris C et al. A comparison of alternative models for the demand for medical care. J Bus Eco Stat 1983;1:115-126.

20. Langa KM, Chernew ME, Kabeto MU et al. The explosion in paid home health care in the 1990s: Who received the additional services? Med Care 2001; 39:147-157.

21. Covinsky KE, Newcomer R, Fox P et al. Patient and caregiver characteristics associated with depression in caregivers of patients with dementia. J Gen Intern Med 2003;18:1006-1014.

22. Lacayo C. Current trends in living arrangements and social environment among ethnic minority elderly. In: Standford EP, Torres-Gil FM, eds. Diversity: New Approaches to Ethnic Minority Aging. Amityville: Baywood, 1992, pp 81-98.

23. Liu K, Manton KG, Aragon C. Changes in home care use by disabled elderly persons: 1982-94. J Gerontol B Psychol Sci Soc Sci 2000;55B:S245S253.

24. Haan MN, Mungas DM, Gonzalez HM et al. Prevalence of dementia in older Latinos: The influence of type 2 diabetes mellitus, stroke and genetic factors. J Am Geriatr Soc 2003;51:169-177.

25. Gurland BJ, Wilder DE, Lantigua R et al. Rates of dementia in three ethnoracial groups. Int J Geriatr Psychiatry 1999;14:481-493.

26. Yeo G. Ethnicity and dementia. J Am Geriatr Soc 2001;49:1393-1394.

27. Miller B, McFall S, Campbell RT. Changes in sources of community long-term care among African American and white frail older persons. J Gerontol 1994; 49:S14-S24.

28. Janevic MR, Connell CM. Racial, ethnic, and cultural differences in the dementia caregiving experience: Recent findings. Gerontologist 2001;41: 334-347.

29. Solis JM, Marks G, Garcia M et al. Acculturation, access to care, and use of preventive services by Hispanics: Findings from HHANES 1982-84. Am J Public Health 1990;80(Suppl):11-19. 